
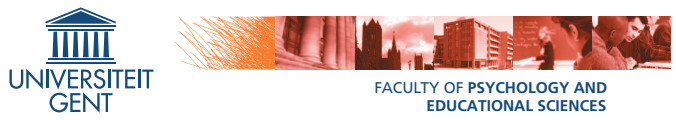

A Joint Modeling Approach for Reaction Time and Accuracy in Psycholinguistic Experiments

\author{
Tom Loeys, Yves Rosseel \& Kristof Baten \\ Department of Data Analysis - Ghent University \\ Tom.Loeys@UGent.be
}

BSS 2010, Spa 


\section{Psycholinguistic Experiment}

Homograph recognition modulated by categorial overlap?

\begin{tabular}{ll}
\hline Condition & Target sentence (homograph / control word) \\
\hline Overlap & She looked up and there seemed to be an ANGEL / ALIEN. \\
No Overlap & He told me he thinks this news is very BIG / SAD. \\
\hline
\end{tabular}

96 experimental trials:

- 16 homographs and 16 controls

- 16 filler sentences and 48 non-words 
- L2-lexical decision task

- homographs and their controls in final position of low-constraint English sentences

- presented through serial visual presentation

- Study 1:

32 highly proficient Dutch-English bilinguals

- Study 2:

31 intermediate proficient Dutch-English bilinguals 


\section{HIGH PROFICIENCY GROUP}
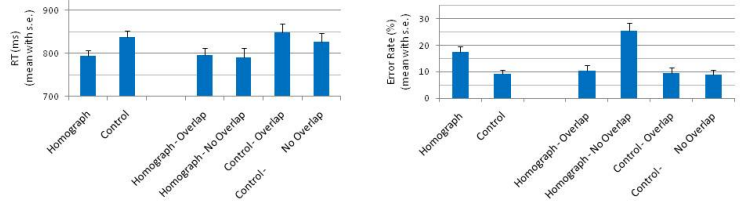

\section{INTERMEDIATE PROFICIENCY GROUP}
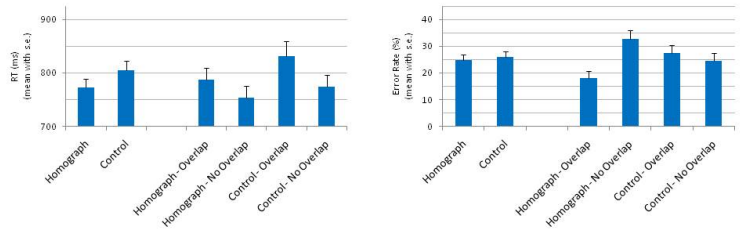

4 


\section{Notation}

- Subjects $i=1, \ldots, N$ and items by $j=1, \ldots, K$

- Two sources of information:

(1) the reaction time $T_{i}=\left(T_{i 1}, \ldots, T_{i K}\right)$

(2) the response accuracy $Y_{i}=\left(Y_{i 1}, \ldots, Y_{i K}\right)$

- $X_{1 i}$ a subject specific characteristic

- $X_{2 j}$ an item specific characteristic 


\section{Analysis of reaction times}

Common approach: F1- and F2-statistics

F1: ANOVA on the mean per participant per item condition

$\Rightarrow$ ignores variability due to items

F2: ANOVA on the mean values per item

Are both significant? 


\section{A better model for the reaction time}

A linear mixed model with crossed random effects:

$$
T_{i j}=\alpha_{0}+\alpha_{1} X_{1 i}+\alpha_{2} X_{2 j}+\theta_{1 i}+\tau_{1 j}+\epsilon_{i j}
$$

$\theta_{1 i}:$ speed (large values=slower responders)

$\tau_{1 j}$ : time intensity (large values=larger reaction times)

with $\epsilon_{i j} \sim N\left(0, \sigma^{2}\right)$ 


\section{Further improvements?}

(i) participant and item variability

(ii) increasing variance with means

(iii) a non-zero minimum

$\Rightarrow$ shifted 3-parameter Weibull distribution for $T_{i j}$

$$
f(t \mid \psi, \lambda, \gamma)=\lambda \gamma(t-\psi)^{\gamma-1} \exp \left[-\lambda(t-\psi)^{\gamma}\right], \quad t \geq \psi
$$

with participant specific shifts $\psi_{i}$ and shapes $\gamma_{i}$ with participant and item specific rate parameter $\lambda_{i j}$

$$
\log \lambda_{i j}=-\tilde{\alpha}_{0}-\tilde{\alpha}_{1} x_{1 i}-\tilde{\alpha}_{2} x_{2 j}-\tilde{\theta}_{1 i}-\tilde{\tau}_{1 j}
$$




\section{A model for the accuracy}

A mixed effects logistic regression model for the probability of incorrect response:

$\operatorname{logit}\left(P\left(Y_{i j}=1\right)\right)=\beta_{0}+\beta_{1} X_{1 i}+\beta_{2} X_{2 j}+\theta_{2 i}+\tau_{2 j}$

$\theta_{2 i}$ : participant's ability

$\tau_{2 j}$ : item's difficulty 


\section{Inspecting the correlations from the separate models...}
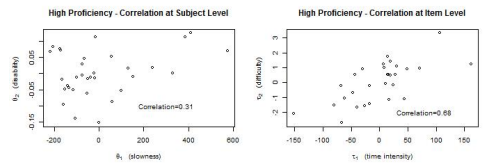

Intermediate Proficiency - Correlation at Subject Level

Intermediate Proficiency - Correlation at Item Level
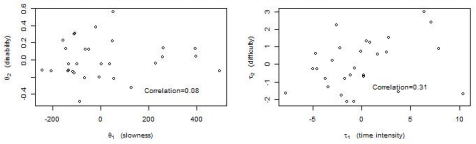

based on normal distribution for RT 


\section{Joint modeling approach}

$\left\{\begin{array}{l}T_{i j}=\alpha_{0}+\alpha_{1} X_{1 i}+\alpha_{2} X_{2 j}+\theta_{1 i}+\tau_{1 j}+\epsilon_{i j} \\ \operatorname{logit}\left(P\left(Y_{i j}=1\right)\right)=\beta_{0}+\beta_{1} X_{1 i}+\beta_{2} X_{2 j}+\theta_{2 i}+\tau_{2 j}\end{array}\right.$

- subjects parameters $\theta_{1 i}$ and $\theta_{2 i}$ follow a bivariate normal distribution with variance-covariance

$$
\Sigma_{S}=\left(\begin{array}{cc}
\sigma_{\theta 1}^{2} & \rho_{\theta} \sigma_{\theta 1} \sigma_{\theta 2} \\
\rho_{\theta} \sigma_{\theta 1} \sigma_{\theta 2} & \sigma_{\theta 2}^{2}
\end{array}\right)
$$

$\Rightarrow \rho_{\theta}$ : correlation between 'speed' and 'ability' 
- item parameters $\tau_{1 i}$ and $\tau_{2 i}$ follow a bivariate normal distribution with variance-covariance

$$
\Sigma_{I}=\left(\begin{array}{cc}
\sigma_{\tau 1}^{2} & \rho_{\tau} \sigma_{\tau 1} \sigma_{\tau 2} \\
\rho_{\tau} \sigma_{\tau 1} \sigma_{\tau 2} & \sigma_{\tau 2}^{2}
\end{array}\right)
$$

$\Rightarrow \rho_{\tau}$ correlation between 'intensity' and 'difficulty'

- similarly in case of shifted Weibull for reaction time distribution

$$
\left\{\begin{array}{l}
\log \lambda_{i j}=-\tilde{\alpha}_{0}-\tilde{\alpha}_{1} x_{1 i}-\tilde{\alpha}_{2} x_{2 j}-\tilde{\theta}_{1 i}-\tilde{\tau}_{1 j} \\
\operatorname{logit}\left(P\left(Y_{i j}=1\right)\right)=\beta_{0}+\beta_{1} X_{1 i}+\beta_{2} X_{2 j}+\theta_{2 i}+\tau_{2 j}
\end{array}\right.
$$




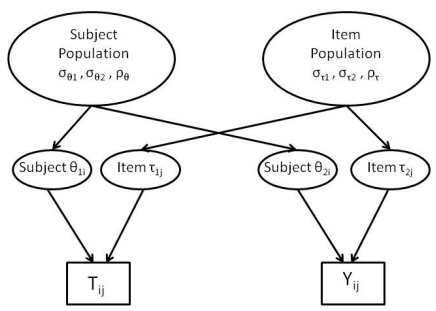

13 


\section{Estimation: a Bayesian approach}

- independent normal distributions with zero mean and large variances for the fixed effect parameters

- the inverse Wishart distribution for the covariance matrix of a multivariate normal distributions,

$$
\Sigma_{S} \sim \text { Inverse - Wishart }\left(\Sigma_{S 0}^{-1}, \kappa_{S 0}\right) \text { and } \Sigma_{I} \sim \text { Inverse - Wishart }\left(\Sigma_{I 0}^{-1}, \kappa_{I 0}\right)
$$

$\Rightarrow$ provides info about the scale of random effects

$$
\begin{aligned}
\kappa & =2: \text { least informative } \\
\Sigma^{-1} & =\omega\left(\begin{array}{ll}
1 & 0 \\
0 & 1
\end{array}\right) \text { informative through } \omega .
\end{aligned}
$$

- $\Gamma\left(\eta_{1}, \eta_{2}\right)$ for measurement precision $1 / \sigma$ 
- in case of shifted Weibull distribution:

$$
\begin{aligned}
& \gamma_{i}: \Gamma\left(\zeta_{1}, \zeta_{2}\right) \text {-distribution } \\
& \psi_{i}: \text { flat uniform distribution }
\end{aligned}
$$

- implemented in WINBUGS (requires 'zeros trick' based on Poisson for shifted Weibull) 


\section{Joint modeling approach: estimated correlations}
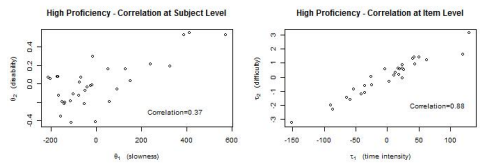

Intermediate Proficiency - Correlation at Subject Level

Intermediate Proficiency - Correlation at Item Level
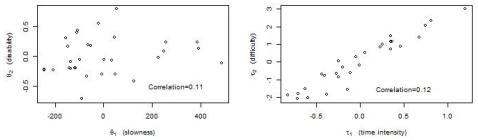

based on normal distribution for RT 


\section{Convergence of covariance parameters at the item level}
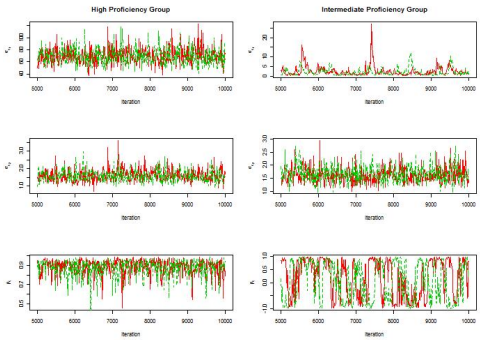

- 2 chains of length 10000

- burn-in period of length 5000 
High Proficiency - Correlation at Subject Level

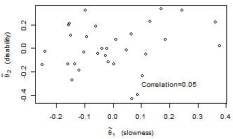

Intermediate Proficiency - Correlation at Subject Leve

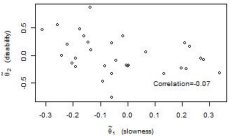

High Proficiency - Correlation at Item Level

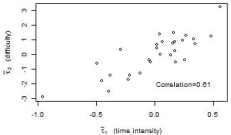

Intermediate Proficiency - Correlation at Item Level

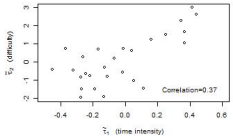

based on shifted Weibull distribution for RT 


\section{Questions?}

- correlations more biased towards 0 for separate modeling?

- maximum likelihood vs. Bayesian framework?

- gain precision for fixed effects when joint modeling?

- criterium to assess need for joint modeling? 


\section{Findings simulation study}

Compare: SEP-B (separate modeling - Bayesian), SEP-M (separate modeling - maximum likelihood) JOINT (joint modeling - Bayesian framework)

- little overall difference between SEP-B and SEP-M

- SEP-B and SEP-M tend to give more biased estimates towards zero for correlations $\rho_{\theta}$ and $\rho_{\tau}$ compared to JOINT

- increasing the number of subjects or increasing the item variability on reaction time and accuracy resulted in less biased estimates of the item correlation $\rho_{\tau}$, but with JOINT still outperforming SEP-B and SEP-M 
- similar findings for the subject correlation are observed when the number of items or the subject variability are increased

- while some gain in efficiency for the fixed effect parameters could be expected from JOINT we did not see such effects

- DIC favored the JOINT above SEP-B with a difference of at least 3 in $97.6 \%, 25.6 \%$ and $4.4 \%$ of the cases when correlations were high, low and zero, respectively (DIC: mimics AIC: looks at the posterior expectation of the deviance function) 


\section{Future research}

- 'censoring' totally ignored so far

- correlation in other psycholinguistic experiments

- diffusion versus hierarchical models 